International Mathematical Forum, 1, 2006, no. 35, 1733-1742

\title{
Global Robust Stability for Neural Networks with Delays
}

\author{
Ju H. Park \\ Department of Electrical Engineering \\ Yeungnam University \\ 214-1 Dae-Dong, Kyongsan 712-749, Republic of Korea
}

\begin{abstract}
The problem of global robust stability of neural networks with time delays and uncertainties is investigated. The uncertainties are assumed to be norm-bounded. The problem is discussed based on the Lyapunov method and linear matrix inequality (LMI) techniques. A novel criterion is given to ascertain the robust stability of the system. The criterion is expressed in terms of LMIs. It is computationally efficient, since the LMIs can be easily solvable by various convex optimization algorithms.
\end{abstract}

Mathematics Subject Classification: 34D23, 93D09, 93D20

Keywords: Robust stability, Lyapunov method, Delays; Neural networks, LMI

\section{Introduction}

In the past decades, various aspects of different neural networks such as Hopfield neural networks, cellular neural networks, Lotka-Volterra neural networks, and bidirectional associative memory neural networks have received a great deal of interest due to their extensive applications in the fields of signal processing, pattern recognition, fixed-point computation, optimization and associative memories, and so on (see [1-5] and the references cited therein).

On the other hand, time delay is likely to be present due to the finite switching speed of amplifiers and occurs in signal transmission among the cells in the electronic implementation of neural networks. Its existence is frequently a source of oscillation and instability. Thus, the stability analysis of delayed neural networks (DNN) have been studied by many researchers in recent years [3-5]. In practice, the weight coefficients of the neurons depend on certain resistance and capacitance values which are subject to uncertainties. For this 
reason, it is important to ensure that system be stable with respect to these uncertainties. Several results related on robust stability have been reported in the literature [6-10].

In this paper, we deal with the problem of robust stability analysis for DNN in which the values of the parameters are not exactly known but bounded in magnitude. Based on the Lyapunov theory and LMI technique, a novel robust stability criterion is given in terms of two LMIs. The advantage of the proposed approach is that resulting stability criterion can be performed efficiently via existing convex optimization algorithms for solving the LMIs [11].

\section{Preliminaries}

Throughout the paper, $\mathcal{R}^{n}$ denotes the $n$ dimensional Euclidean space, and $\mathcal{R}^{n \times m}$ is the set of all $n \times m$ real matrices. $I$ denotes the identity matrix with appropriate dimensions. $\|\cdot\|$ denotes the Euclidean norm of given vector. * denotes the elements below the main diagonal of a symmetric block matrix. $\operatorname{diag}\{\cdots\}$ denotes the block diagonal matrix. For symmetric matrices $X$ and $Y$, the notation $X>Y$ (respectively, $X \geq Y$ ) means that the matrix $X-Y$ is positive definite, (respectively, nonnegative).

The following facts and lemmas will be used for deriving main result.

FACT 1. (Schur complement) Given constant symmetric matrices $\Sigma_{1}, \Sigma_{2}, \Sigma_{3}$ where $\Sigma_{1}=\Sigma_{1}^{T}$ and $0<\Sigma_{2}=\Sigma_{2}^{T}$, then $\Sigma_{1}+\Sigma_{3}^{T} \Sigma_{2}^{-1} \Sigma_{3}<0$ if and only if

$$
\left[\begin{array}{cc}
\Sigma_{1} & \Sigma_{3}^{T} \\
\Sigma_{3} & -\Sigma_{2}
\end{array}\right]<0, \text { or }\left[\begin{array}{cc}
-\Sigma_{2} & \Sigma_{3} \\
\Sigma_{3}^{T} & \Sigma_{1}
\end{array}\right]<0
$$

FACT 2. For any positive scalar $\epsilon$ and vectors $x$ and $y$, the following inequality holds:

$$
x^{T} y+y^{T} x \leq \epsilon x^{T} x+\epsilon^{-1} y^{T} y .
$$

Lemma 1. [14] Consider an operator $\mathcal{D}(\cdot): \mathcal{C}_{n, h} \rightarrow \mathcal{R}^{n}$ with $\mathcal{D}\left(x_{t}\right)=$ $x(t)+\hat{B} \int_{t-h}^{t} x(s) d s$, where $x(t) \in \mathcal{R}^{n}$ and $\hat{B} \in \mathcal{R}^{n \times n}$. For a given scalar $\delta$, where $0<\delta<1$, if a positive definite symmetric matrix $M$ exists, such that

$$
\left[\begin{array}{cc}
-\delta M & h \hat{B}^{T} M \\
h M \hat{B} & -M
\end{array}\right]<0
$$

holds, then the operator $\mathcal{D}\left(x_{t}\right)$ is stable.

LEMma 2. [13] For any constant matrix $\Sigma \in \mathcal{R}^{n \times n}, \Sigma=\Sigma^{T}>0$, scalar $\gamma>0$, vector function $\omega:[0, \gamma] \rightarrow \mathcal{R}^{n}$ such that the integrations concerned are well defined, then

$$
\left(\int_{0}^{\gamma} \omega(s) d s\right)^{T} \Sigma\left(\int_{0}^{\gamma} \omega(s) d s\right) \leq \gamma \int_{0}^{\gamma} \omega^{T}(s) \Sigma \omega(s) d s
$$




\section{Main results}

Consider the following delayed cellular neural network:

$$
\begin{aligned}
\dot{y}(t)= & -(A+\Delta A(t)) y(t)+(W+\Delta W(t)) f(y(t)) \\
& +\left(W_{1}+\Delta W_{1}(t)\right) f(y(t-h))+J,
\end{aligned}
$$

where $y(t)=\left[y_{1}(t), \cdots, y_{n}(t)\right]^{T} \in \mathcal{R}^{n}$ is the state vector, $f(y(t))=\left[f_{1}\left(y_{1}(t)\right), \cdots\right.$, $\left.f_{n}\left(y_{n}(t)\right)\right]^{T} \in \mathcal{R}^{n}$ is the activation functions, $f(y(t-h))=\left[f_{1}\left(y_{1}(t-h)\right), \cdots\right.$, $\left.f_{n}\left(y_{n}(t-h)\right)\right]^{T} \in \mathcal{R}^{n}, A=\operatorname{diag}\left(a_{i}\right), a_{i}>0$ (a positive diagonal matrix), $W$ and $W_{1}$ are feedback matrices, $J=\left[J_{1}, \cdots, J_{n}\right]^{T}$ is a constant input vector, $h>0$ is any time delay, and $\Delta A(t), \Delta W(t)$ and $\Delta W_{1}(t)$ are parametric uncertainties. The time-varying uncertainties are assumed of the form:

$$
\Delta A(t)=H_{0} F_{0}(t) E_{0}, \quad \Delta W(t)=H_{1} F_{1}(t) E_{1}, \Delta W_{1}(t)=H_{2} F_{2}(t) E_{2},
$$

where $H_{i}$ and $E_{i}(i=0,1,2)$ are known constant matrices of appropriate dimensions, and $F_{0}(t), F(t)$ and $F_{2}(t)$ are unknown time-varying matrices with Lebesgue measurable elements bounded by $F_{i}^{T}(t) F_{i}(t) \leq I$ for $i=0,1,2$.

Since $f_{i}, i=1,2, \cdots, n$ are bounded, by using the well-known Brouwer's fixed point theorem, one can easily prove that there exists at least one equilibrium point for Eq. (3) [6].

Assume $y^{*}=\left(y_{1}^{*}, y_{2}^{*}, \cdots, y_{n}^{*}\right)^{T}$ is an equilibrium point of the Eq. (3), then the transformation $x_{i}=y_{i}-y_{i}^{*}$ transforms (3) into the following system:

$$
\begin{aligned}
\dot{x}(t)= & -(A+\Delta A(t)) x(t)+(W+\Delta W(t)) g(x(t)) \\
& +\left(W_{1}+\Delta W_{1}(t)\right) g(x(t-h)),
\end{aligned}
$$

where $g(x)=\left[g_{1}(x), \cdots, g_{n}(x)\right]^{T}$ and $g_{j}\left(x_{j}(t)\right)=f_{j}\left(x_{j}(t)+y_{j}^{*}\right)-f_{j}\left(y_{j}^{*}\right)$ with $g_{j}(0)$. As in many applications, one can assume that each activation function $g(x)$ satisfies the following sector condition:

$$
g_{j}\left(x_{j}(t)\right)\left(g_{j}\left(x_{j}(t)\right)-k x_{j}(t)\right) \leq 0, \quad j=1,2, \cdots, n,
$$

where $k \in \mathcal{R}^{+}$.

Now, to derive less conservative stability criteria for the system (5), define a new mathematical operator $\mathcal{D}\left(x_{t}\right): \mathcal{C}_{n, h} \rightarrow \mathcal{R}^{n}$ as

$$
\mathcal{D}\left(x_{t}\right)=x(t)+\int_{t-h}^{t} G x(s) d s,
$$

where $x_{t}=x(t+s), s \in[-h, 0]$ and $G$ is a weighting matrix of appropriate dimension to be determined later.

From the definition of $\mathcal{D}\left(x_{t}\right)$, the transformed system is

$$
\begin{aligned}
\dot{\mathcal{D}}\left(x_{t}\right)= & -(A+\Delta A(t)-G) x(t)+(W+\Delta W(t)) g(x(t)) \\
& +\left(W_{1}+\Delta W_{1}(t)\right) g(x(t-h))-G x(t-h) .
\end{aligned}
$$


Now we will present a new result for robust stability of Eq. (3).

Theorem 1: For given $h$, the equilibrium point of Eq. (3) is globally asymptotically stable if there exist positive definite matrices $P, S, Q, D=$ $\operatorname{diag}\left\{d_{1}, \cdots, d_{n}\right\}$, a matrix $Y$, and positive scalars $\epsilon_{i},(i=1,2, \cdots, 6)$ satisfying the following two LMIs:

$$
\left[\begin{array}{ccccccccc}
\Pi_{1} & \Pi_{2} & -Y & P W & 0 & P W_{1} & 0 & \Pi_{6} & 0 \\
\star & \Pi_{3} & 0 & 0 & 0 & 0 & 0 & 0 & 0 \\
\star & \star & -S & 0 & 0 & 0 & 0 & -Y^{T} & 0 \\
\star & \star & \star & \Pi_{4} & E_{1}^{T} & D W_{1} & 0 & W^{T} P & 0 \\
\star & \star & \star & \star & -I & 0 & 0 & 0 & 0 \\
\star & \star & \star & \star & \star & \Pi_{5} & E_{2}^{T} & W_{1}^{T} P & 0 \\
\star & \star & \star & \star & \star & \star & -I & 0 & 0 \\
\star & \star & \star & \star & \star & \star & \star & -P & \Pi_{7} \\
\star & \star & \star & \star & \star & \star & \star & \star & \Pi_{8}
\end{array}\right]<0,
$$

where

$$
\begin{aligned}
& \Pi_{1}=-P A-A^{T} P+Y+Y^{T}+S+\left(\epsilon_{1}+\epsilon_{4}\right) E_{0}^{T} E_{0}, \\
& \Pi_{2}=\left[\begin{array}{lllll}
h Y^{T} & P H_{0} & E_{0}^{T} & P H_{1} & P H_{2}
\end{array}\right] \text {, } \\
& \Pi_{3}=\operatorname{diag}\left\{-P,-\epsilon_{1} I,-I,-\epsilon_{2} I,-\epsilon_{3} I\right\} \text {, } \\
& \Pi_{4}=-(2 / k) D A+Q+D W+W^{T} D+D H_{0} H_{0}^{T} D \\
& +\epsilon_{2} E_{1}^{T} E_{1}+D H_{1} H_{1}^{T} D+D H_{2} H_{2}^{T} D+\epsilon_{5} E_{1}^{T} E_{1} \text {, } \\
& \Pi_{5}=-Q+\left(\epsilon_{3}+\epsilon_{6}\right) E_{2}^{T} E_{2}, \quad \Pi_{6}=-A^{T} P+Y^{T}, \\
& \Pi_{7}=\left[\begin{array}{lll}
P H_{0} & P H_{1} & P H_{2}
\end{array}\right], \Pi_{8}=\operatorname{diag}\left\{-\epsilon_{4} I,-\epsilon_{5} I,-\epsilon_{6} I\right\} .
\end{aligned}
$$

Proof. For $P>0, Q>0, S>0$ and a positive scalar $d_{i}$, consider the positive definite Lyapunov functional

$$
V=V_{1}+V_{2}+V_{3}+V_{4}+V_{5}
$$

where

$$
\begin{aligned}
V_{1} & =\mathcal{D}^{T}\left(x_{t}\right) P \mathcal{D}\left(x_{t}\right), \quad V_{2}=2 \sum_{i=1}^{n} d_{i} \int_{0}^{x_{i}(t)} g_{i}(s) d s \\
V_{3} & =\int_{t-h}^{t} g^{T}(x(s)) Q g(x(s)) d s, \quad V_{4}=h \int_{t-h}^{t} \int_{s}^{t} x^{T}(s) G^{T} P G x(s) d u d s, \\
V_{5} & =\int_{t-h}^{t} x^{T}(s) S x(s) d s .
\end{aligned}
$$


The time derivative of $V$ along the trajectory of Eq. (5) and (8) is

$$
\begin{aligned}
\dot{V}= & 2(x(t)+\xi(t))^{T} P(-(A+\Delta A(t)-G) x(t)+(W+\Delta W) g(x(t)) \\
& \left.+\left(W_{1}+\Delta W_{1}\right) g(x(t-h))-G x(t-h)\right)+2 g^{T}(x(t)) D[-(A+\Delta A(t)) x(t) \\
& \left.+(W+\Delta W) g(x(t))+\left(W_{1}+\Delta W_{1}\right) g(x(t-h))\right]+g^{T}(x(t)) Q g(x(t)) \\
& -g^{T}(x(t-h)) Q g(x(t-h))+h^{2} x^{T}(t) G^{T} P G x(t) \\
& -h \int_{t-h}^{t} x^{T}(s) G^{T} P G x(s) d s+x^{T}(t) S x(t)-x^{T}(t-h) S x(t-h),
\end{aligned}
$$

where $\xi(t)=\left(\int_{t-h}^{t} G x(s) d s\right)$.

Here note that

$$
\begin{aligned}
& -2 g^{T}(x(t)) D A x(t) \leq-\frac{2}{k} g^{T}(x(t)) D A g(x(t)), \\
& -h \int_{t-h}^{t} x^{T}(s) G^{T} P G x(s) d s \leq-\xi^{T}(t) P \xi(t),
\end{aligned}
$$

where Lemma 2 is used in second inequality.

Using Eqs. (13) and (14), then we have the following inequality:

$$
\dot{V} \leq z^{T}\left[\begin{array}{ccccc}
M_{1} & -P G & M_{3} & M_{5} & M_{8} \\
\star & -S & 0 & 0 & -G^{T} P \\
\star & \star & M_{2} & M_{4} & M_{7} \\
\star & \star & \star & -Q & M_{6} \\
\star & \star & \star & \star & -P
\end{array}\right] z \equiv z^{T} \mathcal{M} z
$$

where $M_{1}=-P(A+\Delta A(t)-G)-(A+\Delta A(t)-G)^{T} P+h^{2} G^{T} P G+S$, $M_{2}=-(2 / k) D A+Q+D(W+\Delta W(t))+(W+\Delta W(t))^{T} D, M_{3}=P(W+$ $\Delta W(t))-\Delta A^{T} D, M_{4}=D\left(W_{1}+\Delta W_{1}(t)\right), M_{5}=P\left(W_{1}+\Delta W_{1}(t)\right), M_{6}=$ $\left(W_{1}+\Delta W_{1}(t)\right)^{T} P, M_{7}=(W+\Delta W(t))^{T} P, M_{8}=-(A+\Delta A(t)-G)^{T} P$, and

$$
z=\left[\begin{array}{lllll}
x^{T}(t) & x^{T}(t-h) & g^{T}(x(t)) & g^{T}(x(t-h)) & \xi^{T}(t)
\end{array}\right]^{T} .
$$

If the matrix $\mathcal{M}$ is negative definite, $\dot{V}<0$. Here, let us define $Y$ as $Y=P G$. Then, applying Fact 2 to the terms containing uncertain factor in $\mathcal{M}$ gives that

$$
\begin{aligned}
& \quad-2 x^{T}(t) P \Delta A(t) x(t) \leq \epsilon_{1}^{-1} x^{T}(t) P H_{0} H_{0}^{T} P x(t)+\epsilon_{1} x^{T}(t) E_{0}^{T} E_{0} x(t), \\
& 2 x^{T}(t) P \Delta W(t) g(x(t)) \leq \epsilon_{2}^{-1} x^{T}(t) P H_{1} H_{1}^{T} P x(t)+\epsilon_{2} g^{T}(x(t)) E_{1}^{T} E_{1} g(x(t)), \\
& 2 x^{T}(t) P \Delta W_{1}(t) g(x(t-h)) \leq \epsilon_{3}^{-1} x^{T}(t) P H_{2} H_{2}^{T} P x(t) \\
& \quad \quad+\epsilon_{3} g^{T}(x(t-h)) E_{2}^{T} E_{2} g(x(t-h)), \\
& -2 x^{T}(t) \Delta A^{T}(t) P \xi(t) \leq \epsilon_{4} x^{T}(t) E_{0}^{T} E_{0} x(t)+\epsilon_{4}^{-1} \xi(t)^{T} P H_{0} H_{0}^{T} P \xi(t), \\
& -2 x^{T}(t) \Delta A^{T}(t) D g(x(t)) \leq g^{T}(x(t)) D H_{0} H_{0}^{T} D g(x(t))+x^{T}(t) E_{0}^{T} E_{0} x(t),
\end{aligned}
$$




$$
\begin{aligned}
& 2 g^{T}(x(t)) D \Delta W(t) g(x(t)) \leq g^{T}(x(t)) D H_{1} H_{1}^{T} D g(x(t)) \\
& \quad+g^{T}(x(t)) E_{1}^{T} E_{1} g(x(t)), \\
& 2 g^{T}(x(t)) D \Delta W_{1}(t) g(x(t-h)) \leq g^{T}(x(t)) D H_{2} H_{2}^{T} D g(x(t)) \\
& \quad+g^{T}(x(t-h)) E_{2}^{T} E_{2} g(x(t-h)), \\
& 2 g^{T}(x(t)) \Delta W^{T}(t) P \xi(t) \leq \epsilon_{5} g^{T}(x(t)) E_{1}^{T} E_{1} g(x(t))+\epsilon_{5}^{-1} \xi(t)^{T} P H_{1} H_{1}^{T} P \xi(t), \\
& 2 g^{T}(x(t-h)) \Delta W_{1}^{T}(t) P \xi(t) \leq \epsilon_{6}^{-1} \xi(t)^{T} P H_{2} H_{2}^{T} P \xi(t) \\
& \quad+\epsilon_{6} g^{T}(x(t-h)) E_{2}^{T} E_{2} g(x(t-h)),
\end{aligned}
$$

where $\epsilon_{i}, i=1,2, \cdots, 6$ is the positive scalars.

Using the relationship (16), we have a new upper bound of $\dot{V}$ as

$$
\dot{V} \leq z^{T}\left[\begin{array}{ccccc}
N_{1} & -Y & P W & P W_{1} & -A^{T} P+Y^{T} \\
\star & -S & 0 & 0 & -Y^{T} \\
\star & \star & N_{2} & D W_{1} & W^{T} P \\
\star & \star & \star & N_{3} & W_{1}^{T} P \\
\star & \star & \star & \star & N_{4}
\end{array}\right] z \equiv z^{T} \mathcal{N} z
$$

where

$$
\begin{aligned}
N_{1}= & -P A-A^{T} P+Y+Y^{T}+h^{2} Y^{T} P^{-1} Y+S+\epsilon_{1}^{-1} P H_{0} H_{0}^{T} P+\epsilon_{1} E_{0}^{T} E_{0} \\
& +E_{0}^{T} E_{0}+\epsilon_{2}^{-1} P H_{1} H_{1}^{T} P+\epsilon_{3}^{-1} P H_{2} H_{2}^{T} P+\epsilon_{4} E_{0}^{T} E_{0}, \\
N_{2}= & -(2 / k) D A+Q+D W+W^{T} D+D H_{0} H_{0}^{T} D+\epsilon_{2} E_{1}^{T} E_{1}+D H_{1} H_{1}^{T} D \\
& +E_{1}^{T} E_{1}+D H_{2} H_{2}^{T} D+\epsilon_{5} E_{1}^{T} E_{1}, \\
N_{3}= & -Q+\epsilon_{3} E_{2}^{T} E_{2}+E_{2}^{T} E_{2}+\epsilon_{6} E_{2}^{T} E_{2}, \\
N_{4}= & -P+\epsilon_{4}^{-1} P H_{0} H_{0}^{T} P+\epsilon_{5}^{-1} P H_{1} H_{1}^{T} P+\epsilon_{6}^{-1} P H_{2} H_{2}^{T} P .
\end{aligned}
$$

By Fact 1, the inequality $\mathcal{N}<0$ is equivalent to the LMI (9). Also, the inequality (10) is equivalent to

$$
\left[\begin{array}{cc}
-P & h G^{T} P \\
\star & -P
\end{array}\right]<0 .
$$

If the above inequality holds, then we can prove that a positive scalar $\delta$ which is less than one exists such that

$$
\left[\begin{array}{cc}
-\delta P & h G^{T} P \\
\star & -P
\end{array}\right]<0
$$

according to matrix theory. Therefore, from Lemma 1, if the inequality (10) holds, then operator $\mathcal{D}\left(x_{t}\right)$ is stable. According to the Theorem 9.8.1 in [15], we conclude that if LMIs (9)-(10) hold, then, the equilibrium point of system (3) is asymptotically stable. This completes our proof. 
REMARK 1: The criterion given in Theorem 1 is dependent on the time delay $h$. It is well known that the delay-dependent criteria are less conservative than delay-independent criteria when the delay is small. By iteratively solving the matrix inequalities given in Theorem 1 with respect to $h$, one can find the maximum allowable upper bound $\bar{h}$ of time delay $h$ for guaranteeing robust stability of system (3). The solutions of Theorem 1 can be obtained by solving the eigenvalue problem with respect to solution variables, which is a convex optimization problem [11]. In this paper, we utilize Matlab's LMI Control Toolbox [12] which implements interior-point algorithm. This algorithm is significantly faster than classical convex optimization algorithms [11].

Remark 2: From the solutions $P$ and $Y$ of the problem given in Theorem 1 , the matrix $G$ in Eq. (7) is obtained from the relation $G=P^{-1} Y$. In the work of Singh [8], robust stability of DNN with $A=I$ and $\Delta A(t)=0$ has been studied. Thus it is a special case of our work.

\section{Numerical examples}

EXAmple 1: In this example, we compare the existing delay-dependent criteria [3, 7] with ours. Consider a nominal DNN (3) $(\Delta A(t)=\Delta W(t)=$ $\left.\Delta W_{1}(t)=0\right)$ studied in $[3,7]$ with:

$$
A=\left[\begin{array}{cc}
0.7 & 0 \\
0 & 0.7
\end{array}\right], \quad W=0, \quad W_{1}=\left[\begin{array}{ll}
0.1 & 0.1 \\
0.3 & 0.3
\end{array}\right],
$$

and the activation function $g_{i}(x)=0.5(|x+1|-|x-1|)$.

The existing delay-dependent criteria provide the maximum allowable bound $\bar{h}$ guaranteeing the stability of the system as $\bar{h}=0.3638$ [7] and $\bar{h}=0.45$ [3], respectively. On the other hand, for the given $g_{i}(x)$, let us take the parameter $k$ as $k=1.01$. By iteratively applying our criterion given in Theorem 1 , it is easy to verify that we have the maximum allowable bound, $\bar{h}=\infty$. It means that for this example our criteria show that the system is actually delay-independent stable, which is not possible to know from the existing delay-dependent criteria [3, 7].

EXAMPLE 2: Consider a nominal DNN (3) $\left(\Delta A(t)=\Delta W(t)=\Delta W_{1}(t)=\right.$ 0) with:

$$
A=I, \quad W=\left[\begin{array}{cc}
0 & 0.9 \\
-1 & -1
\end{array}\right], \quad W_{1}=\left[\begin{array}{cc}
0.6 & -1 \\
1 & \mu
\end{array}\right],
$$

and the activation function $g_{i}(x)=0.5(|x+1|-|x-1|)$.

In this example, we compare the existing delay-independent criteria with ours 
by determining $\mu$ for guaranteeing stability of the system.

First, it is easy to verify that the criterion $\left(W+W^{T}<0, \quad\left\|W_{1}\right\| \leq 1\right)$ by Arik and Tavsanoglu [4] is not applicable since $W+W^{T} \nless 0$. Secondly, the criterion $\left(-D+D W+W^{T} D+W_{1}^{T} D W_{1}<0\right)$ by Singh [8] guarantees the stability for the interval $-0.259 \leq \mu \leq 0.766$. Finally, Theorem 1 guarantees the stability when $-1.99 \leq \mu \leq 1.559$ for any delay $h>0$.

EXAMPLE 3: Consider the following uncertain DNN:

$$
\begin{aligned}
\dot{x}(t)= & -\left(A+H_{0} F_{0}(t) E_{0}\right) x(t)+\left(W+H_{1} F_{1}(t) E_{1}\right) g(x(t)) \\
& +\left(W_{1}+H_{2} F_{2}(t) E_{2}\right) g(x(t-h)),
\end{aligned}
$$

where

$$
\begin{aligned}
& A=I, \quad W=\left[\begin{array}{cc}
0.4 & 0.1 \\
0.1 & -0.5
\end{array}\right], \quad W_{1}=0.3 I, H_{0}=0, \quad H_{1}=H_{2}=0.2 I, \\
& E_{0}=0, \quad E_{1}=E_{2}=I, g_{i}(x)=0.5(|x+1|-|x-1|), \quad F_{i}^{T}(t) F_{i}(t) \leq I, \forall i .
\end{aligned}
$$

First, let's apply the stability criteria given by Singh [8], Zhang et al. [9], and Rong [10] to system (20). Then, one can see that all the criteria are not satisfied.

Next, let's apply our result, Theorem 1, to the system (20). Then, it can be easily verified that the LMIs given in Theorem 1 are feasible for any time delay $h>0$.

\section{Concluding remarks}

A novel criterion for the global robust stability of uncertain DNN has been presented by the Lyapunov theory and linear matrix inequality framework. The criterion is delay-dependent and expressed by two LMIs. To show the effectiveness of the proposed criterion, three numerical examples are given. The criterion turns out to be an improvement over the criteria in the literature.

\section{Acknowledgements}

This work was supported in part by the Korea Research Foundation Grant funded by Korean Government (MOEHRD) (KRF-2005-041-D00280). 


\section{References}

[1] L.O. Chua and L. Yang, "Cellular neural networks: theory and applications," IEEE Trans. Circuits Syst. I, Fundam. Theory Appl., vol. 35, pp.1257-1290, 1988.

[2] B. Xu, X. Liu, and X. Liao, "Global asymptotic stability of high-order Hopfield type neural networks with time delays," Computers and Mathematics with Applications, vol. 45, pp.1729-1737, 2003.

[3] Q. Zhang, X. Wei, and J. Xu, "Global asymptotic stability of Hopfield neural networks with transmission delays," Physics Letters A, vol. 318, pp.399-405, 2003.

[4] S. Arik, and V. Tavsanoglu, "On the global asymptotic stability of delayed cellular neural networks," IEEE Transactions on Circuits and Systems, Part I: Fundamental Theory and Applications, vol. 47, pp.571-574, 2000.

[5] S. Arik, "Global asymptotic stability of a larger class of neural networks with constant time delay," Physics Letters A, vol. 311, pp.504-511, 2003.

[6] J. Cao, "Global asymptotic stability of neural networks with transmission delays," International Journal of Systems Science, vol. 31, pp.1313-1316, 2000 .

[7] A. Chen, J. Cao, and L. Huang, "An estimation of upperbound of delays for global asymptotic stability of delayed Hopfield neural networks," IEEE Transactions on Circuits and Systems, Part I: Fundamental Theory and Applications, vol. 49 pp. 1028-1032, 2002.

[8] V. Singh, Robust stability of cellular neural networks with delay: linear matrix inequality approach. IEE Proceedings Control Theory and Applications. 2004;151:125-129.

[9] H. Zhang, C. Li, X. Liao, "A note on the robust stability of neural networks with time delay", Chaos, Solitons, \& Fractals, vol. 25, pp.357-360, 2005.

[10] L. Rong, "LMI-based criteria for robust stability of Cohen?Grossberg neural networks with delay ," Physics Letters A, vol. 339, pp.63-73, 2005.

[11] S. Boyd, L.E. Ghaoui, E. Feron, and V. Balakrishnan, Linear Matrix Inequalities in Systems and Control Theory, Studies in Applied Mathematics, SIAM, Philadelphia, Vol. 15, 1994.

[12] P. Gahinet, A. Nemirovski, A. Laub, and M. Chilali, LMI Control Toolbox User's Guide. The Mathworks, Natick, Massachusetts, 1995. 
[13] K. Gu, "An integral inequality in the stability problem of time-delay systems", in Proceedings of IEEE Conf. Decision Control, Sydney, Australia, December 2000, pp. 2805-2810.

[14] D. Yue and S. Won, "Delay-dependent robust stability of stochastic systems with time delay and nonlinear uncertainties", Electronics Letters, Vol. 37, pp.992-993, 2001.

[15] J. Hale and S.M. Verduyn Lunel, Introduction to Functional Differential Equations, Springer-Verlag, New York., 1993.

Received: April 4, 2006 